\title{
Effect of dietary supplementation of Saccharomyces cerevisiae and a postbiotic from Lactobacillus acidophilus on the concentration of organic acids, biogenic amines, and microbiota in the small intestine and colon of rosé veal calves
}

\author{
M. Thorsteinsson' ${ }^{1}$ N. Canibe and M. Vestergaard \\ Aarhus University, Department of Animal Science, Blichers Allé 20, 8830 Tjele, Denmark
}

KEY WORDS: probiotic, Saccharomyces cerevisiae, postbiotic, Lactobacillus acidophilus, calves, microbiota

Received: 11 August 2020

Revised: 20 October 2020

Accepted: 1 December 2020
${ }^{1}$ Corresponding author: e-mail: mitho@anis.au.dk

\begin{abstract}
The effects of the product 'ZooLac Bovimix' on the gastrointestinal tract of rosé veal calves before weaning were investigated. The product contained live yeast (Saccharomyces cerevisiae) and a postbiotic from Lactobacillus acidophilus. Twelve calves were randomly allocated to a control diet (CON; $20.8 \pm 1.5$ days of age and $53.8 \pm 3.8 \mathrm{~kg}$ body weight) and twelve calves were allocated to a treatment supplemented with the product in the milk replacer and concentrate (PRO; $23.7 \pm 1.8$ days of age and $52.8 \pm 0.9 \mathrm{~kg}$ body weight) for three weeks. The CFUs of yeast were $7.88 \times 10^{9}$ and $2.38 \times 10^{9}$ per kg milk replacer and concentrate as feed, respectively. The number of some microbiota groups, concentration of organic acids, and biogenic amines in digesta from the mid-small intestine and mid-colon were measured. In PRO group significantly higher numbers of yeast cells in both the small intestine and colon in comparison to CON group were noted. Additionally, PRO group tended $(P=0.06)$ to have a lower number of Clostridium perfringens in the colon. In both the small intestine and colon, concentrations of the individual and sum of organic acids were unaffected by the treatment. The concentrations of the individual biogenic amines in the small intestine and colon were also unaffected by the treatments. However, PRO group tended to have a lower total concentration of biogenic amines in the colon $(P=0.06)$. So, the supplementation of the product had only minor effects on the gastrointestinal tract of pre-weaned rosé veal calves.
\end{abstract}

\section{Introduction}

Diarrhoea is a major cause of morbidity and mortality in young calves. Additionally, incidences of diarrhoea negatively affect the growth performance and increase the veterinary, feed, and labour costs (Cho and Yoon, 2014). The treatment of diarrhoea in calves often involves the use of antibiotics. The antibiotic therapy in animal production can constitute a threat to human health as it increases the risk of developing multiple antibiotic-resistant bacteria (Baynes et al., 2016). Therefore, strategies to maintain the animal health with a reduced use of antibiotics are searched for.

Manipulation of the gastrointestinal tract (GIT) microbiota through supplementation of feed additives, such as pro- and postbiotics, is an attractive approach to improve and maintain animal health (Fomenky et al., 2017), and thus, lower the use of antibiotics in animal production. Probiotics are live non-pathogenic microorganisms that, when administered in adequate amounts, enhance the 
health of the host by improving the balance in the GIT (Williams, 2010; Martín and Langella, 2019). Several mechanisms have been proposed by which probiotics can exert their action: enhance the innate immunity, disturb adhesion of pathogens, promote intestinal epithelial survival, improve protective intestinal responses and barrier function, and/or decrease pathogen-induced inflammation (Williams, 2010). Saccharomyces cerevisiae (SC) is one of the commonly included probiotics in the diet of production animals; and SC has been found to lower the frequency of diarrhoea in calves (Alugongo et al., 2017). In the GIT, the supplementation of SC has been found to increase the number of living yeast cells in calves (Fomenky et al., 2017) and inhibit the growth of enterobacteria in weaned piglets (Trckova et al., 2014; Zhu et al., 2017).

Postbiotics are non-viable bacterial products or metabolic products from microorganisms that have biological activity in the host (Martín and Langella, 2019). Postbiotics are believed to function as bioactive compounds which may promote animal health (Wegh et al., 2019). Postbiotics from Lactobacillus spp. have shown to increase the number of lactic acid bacteria in the GIT of weaned piglets (Thu et al., 2011; Loh et al., 2013). However, the literature reporting effects of pro- and postbiotic in the GIT, especially in the small intestine and colon, of calves is highly limited.

So, the aim of the current study was to investigate the effects of a product, containing live yeast (SC) and a postbiotic product from Lactobacillus acidophilus, on the microbiota and microbial metabolites in the GIT of pre-weaned rosé veal calves. It was hypothesized that supplementation of the yeast/lactobacillus product would increase the number of living yeast cells and the number of beneficial bacteria in the GIT.

\section{Material and methods}

\section{Animals, housing and diets}

A production trial was conducted at a Danish rosé veal calf operation in Southern Denmark. All procedures involving animals were conducted in accordance with the guidelines of the Danish Ministry of Justice with respect to animal experimentation and care of animals under study (The Danish Ministry of Justice, 2014, LBK no. 474). The Danish Animal Experiments Inspectorate under the Danish Veterinary and Food Administration was consulted for guidance on required permissions and approved the project activities in writing without requiring further formal application and approval process.
On arrival, 120 three-week old bull calves were randomly allocated to either a control diet $(\mathrm{CON}$; 60 calves) or a diet supplemented with a yeast/lactobacillus product in the milk replacer (MR) and concentrate (PRO; 60 calves). The calves arrived in five blocks of 24 calves from November 2018 to January 2019. Each block consisted of two strawbedded hutches which contained two pens with six calves per pen. Before departure from the dairy herds, the calves were weighed. The calves originated from 21 different dairy herds. On arrival at the veal calf operation, the calves were sorted based on their body weight (BW) before departure, breed and dairy herd of origin. Hence, the calves in each pen in the hutch had a similar average BW, proportion of crossbreds and calves per dairy herd. The pens were provided with one trough for concentrate and six bawls where the calves were offered MR and water. Roughage was offered in a shared hay-rack between the two pens in the hutches. Further details of the experimental set-up and recordings, and of the impact of the product on health and growth performance of calves is described by Thorsteinsson and Vestergaard (2020).

Twenty-four of the 120 calves enrolled in the production trial were included in the study on the impact of the product on GIT presented herein.

The calves were fed 61 of MR $(60 \%$ skimmed milk powder) per day and were also offered concentrate and hay ad libitum. Calves allocated to CON group were fed MR and concentrate without any additives, while the PRO group was fed MR supplemented with $2 \mathrm{~g}$ of 'ZooLac Bovimix Milk' (ChemVet; Silkeborg, Denmark) (7.7 MJ net energy (NE)/kg dry matter (DM), $2.83 \%$ crude protein (CP)) per $135 \mathrm{~g}$ of MR). 'ZooLac Bovimix Milk' contains 55\% 'Actisaf Powder' (living SC cells: Lesaffre proprietary strain: NCYC Sc 47/CNCM I-4407) and $45 \%$ 'ZooLac'. 'ZooLac' is produced by fermentation of vinasse by a special strain of $L$. acidophilus which after fermentation is stabilised by thermal treatments, followed by lyophilisation. The final product consists of thermostabilised lactic acid bacteria, lactic acid and lactic acid salts. The number of SC in the MR was $7.88 \pm 0.18 \times 10^{9}$ colony forming unit (CFU) per $\mathrm{kg}$ milk powder (mean $\pm \mathrm{SEM}$ ), and the number of thermostabilised L. acidophilus was $3.1 \times 10^{7}$ per $\mathrm{kg}$ MR.

'Zoolac Bovimix' (ChemVet; Silkeborg, Demark) was added to the PRO concentrate to obtain a concentration of $0.9 \%$ per $\mathrm{kg} \mathrm{DM}(55 \% \mathrm{SC}$ and $45 \%$ 'Zoolac'). Due to the pelleting process, the PRO concentrate contained the yeast product 'Actisaf $\mathrm{HR}^{+}$' from 'Zoolac Bovimix', which contains a more thermostable 
strain of SC in comparison to 'Actisaf Powder' used in 'Zoolac Bovimix Milk'. The CFU of SC in the pelleted PRO concentrate was $2.38 \pm 0.13 \times 10^{9}$ per $\mathrm{kg}$ as feed (mean $\pm \mathrm{SEM}$ ).

At arrival, the average age and $\mathrm{BW}$ were $20.8 \pm 1.5$ days and $53.8 \pm 3.8 \mathrm{~kg}$ for the CON group, and $23.7 \pm 1.8$ days and $52.8 \pm 0.9 \mathrm{~kg}$ for PRO calves, respectively (age: $P=0.20$; $\mathrm{BW}: P=0.92$ ). After three weeks of treatment, eight Holstein calves (four per hutch; two per pen) from three different blocks were randomly chosen and driven to facilities at Aarhus University the day before they were slaughtered. During the three weeks of experiment, none of the 24 calves in total was treated for diarrhoea or any other disease.

At slaughter, CON and PRO groups weighed $71.8 \pm 1.7 \mathrm{~kg}$ and $73.6 \pm 1.5 \mathrm{~kg}$, respectively $(P=0.36)$. From arrival to slaughter, the average daily gain was $838 \mathrm{~g} /$ day and $914 \mathrm{~g} /$ day for $\mathrm{CON}$ and PRO group, respectively $(P=0.21)$. The calves were milk-fed around 7:00, and had access to concentrate, hay and water at all times. The first calf was slaughtered at around 8:30. The eight calves of a block slaughtered on a given day were put down in an order that gave the same average time from the last milk-feeding to slaughter for both treatments. A penetrating captive bolt pistol was used to put the calves down. Afterward, the throat was cut to bleed the calves. The abdomen was incised around 2-3 min after the calf was stunned. The entire GIT was removed from the carcass, and the small intestine and colon were both divided into three equally long segments. Digesta from the 2/3-part of the small intestine and colon were collected and stored at $-20{ }^{\circ} \mathrm{C}$ until they were analysed.

\section{Analytical methods}

For microbial enumeration by plating of digesta from the mid-small intestine and mid-colon, approximately $5 \mathrm{~g}$ were transferred to plastic bags and diluted ten-fold in peptone water (10 g Bacto Peptone (Merck 1.07213)/1 and $1 \mathrm{~g}$ Tween 80/1). The suspension was homogenised in a stomacher blender (Interscience; St. Nom, France) for $2 \mathrm{~min}$. Ten-fold dilutions were prepared in peptone water, and samples $(0.1 \mathrm{ml})$ were plated on selective media. Lactic acid bacteria (LAB) were enumerated on de Man, Rogosa and Sharp (MRS) agar (84607, VWR International, Radnor, PA, USA) following anaerobic incubation at $37{ }^{\circ} \mathrm{C}$ for 2 days. Enterobacteriaceae were enumerated on McConkey agar (84614, VWR International, Radnor, PA, USA) fol- lowing incubation at $37^{\circ} \mathrm{C}$ for 1 day. Yeasts were enumerated on malt chloramphenicol/chlortetracycline (MCA) agar (10 g glucose/1, $3 \mathrm{~g}$ malt extract/l, $3 \mathrm{~g}$ yeast extract/1, $5 \mathrm{~g}$ Bacto Peptone (84687, VWR International, Radnor, PA, USA), 50 mg chlortetracycline $+50 \mathrm{mg}$ chloramphenicol (SR0177E, Oxoid LTD, Basingstoke, Hampshire, England)/l, 15 g agar (84609, VWR International, Radnor, PA, USA)/l) following incubation at $37{ }^{\circ} \mathrm{C}$ for 2 days. Clostridium perfringens were enumerated using the pour-plate technique on Tryptose Sulfite Cycloserine agar (84636, VWR International, Radnor, PA, USA) after anaerobic incubation at $37^{\circ} \mathrm{C}$ for 1 day.

The concentration of organic acids was measured as previously described by Canibe et al. (2007); and biogenic amine concentration (putrescine, cadaverine, and tyramine) was measured according to the procedure described by Poulsen et al. (2018).

\section{Statistical analysis}

In the experimental design, the eight calves randomly picked from two hutches and slaughtered on the same date constituted a block, whereas the hutch acted as a sub-block. Thus, calves, as they arrived over a period of three months in five blocks, were slaughtered from three different blocks and six different sub-blocks.

The data was analysed using a linear mixed model (R, software version 3.5.3, 2019; Boston, MA, USA). The model included the fixed effect of treatment and the random effects of block and sub-block. All data were examined to discard any possible outliers, which were defined as values outside the interval of the mean \pm 3 times standard deviation (see footnotes to Tables). Data were also tested for normality of the residuals by evaluating the QQ-plots constructed in $\mathrm{R}$, and the means were tested for homogeneity of the variance by using Bartlett's test. Statistical significance was declared when $P \leq 0.05$ and statistical tendencies were declared when $0.05<P \leq 0.10$. Results are presented as least squares means and standard error of the mean.

\section{Results}

\section{Microbiota in the small intestine and colon}

The microbiota in the small intestine was not affected by the treatments, except that PRO group had a significantly higher number of yeast cells 
Table 1. Microbiota in the small intestine and colon of rosé veal calves fed a control diet (CON) or a diet supplemented with a product containing yeast (Saccharomyces cerevisiae) and a postbiotic from Lactobacillus acidophilus (PRO) for three weeks*

\begin{tabular}{|c|c|c|c|c|}
\hline Indices & CON & PRO & SEM & $P$-value \\
\hline \multicolumn{5}{|c|}{ Mid-small intestine, log CFU/g } \\
\hline Enterobacteriaceae & $<4.97(1)$ & $<4.73(3)$ & 0.289 & 0.531 \\
\hline lactic acid bacteria & 5.61 & 5.72 & 0.146 & 0.522 \\
\hline Clostridium perfringens & $<2.87(4)$ & $<2.64(6)$ & 0.408 & 0.500 \\
\hline yeast & $<2.88(9)$ & 5.56 & 0.164 & 0.001 \\
\hline \multicolumn{5}{|l|}{ Mid-colon, log CFU/g } \\
\hline Enterobacteriaceae & 7.16 & 6.87 & 0.234 & 0.365 \\
\hline lactic acid bacteria & 7.17 & 6.77 & 0.275 & 0.293 \\
\hline Clostridium perfringens & $<5.71(1)$ & $<5.40(2)$ & 0.169 & 0.064 \\
\hline yeast & $<3.60(6)$ & 4.78 & 0.268 & 0.003 \\
\hline
\end{tabular}

* 24 calves were included in the dataset; 12 calves received CON and 12 calves received PRO diet. Values in parenthesis indicate the number of samples with values below detection levels. The approximate detection levels (log CFU/g) are as follows: Enterobacteriaceae, 4; Clostridium perfringens, 2; yeasts, 3 . The symbol ' $<$ ' indicates that some observations from which the mean was calculated had values below detection levels. When no colonies were detected on the plates, the detection level was applied and used to make the calculations. Thus, the real mean value is lower than that reported. SEM - standard error of the mean

Table 2. Concentration of organic acids in small intestine and colon of rosé veal calves receiving a control diet (CON) or a diet supplemented with a product containing yeast (Saccharomyces cerevisiae) and a postbiotic from Lactobacillus acidophilus (PRO) for three weeks*

\begin{tabular}{lllll}
\hline Indices & CON & PRO & SEM & $P$-value \\
\hline Mid-small intestine, mmol/kg & & & & \\
DL-lactic acid & 4.61 & 4.83 & 0.439 & 0.675 \\
formic acid & 0.16 & 0.16 & 0.067 & 0.985 \\
acetic acid & 0.62 & 0.65 & 0.073 & 0.771 \\
propionic acid & 0.23 & 0.19 & 0.118 & 0.657 \\
butyric acid & 0.01 & 0.00 & 0.006 & 0.302 \\
valeric acid & 0.00 & 0.00 & - & - \\
iso-butyric acid & 0.00 & 0.00 & - & - \\
iso-valeric acid & 0.00 & 0.00 & - & - \\
succinic acid & 0.10 & 0.15 & 0.060 & 0.414 \\
total organic acid & 5.73 & 5.98 & 0.511 & 0.851 \\
Mid-colon, mmol/kg & & & & \\
DL-lactic acid & 0.43 & 0.49 & 0.289 & 0.697 \\
formic acid & 0.30 & 0.16 & 0.178 & 0.323 \\
acetic acid & 42.0 & 43.7 & 4.43 & 0.681 \\
propionic acid & 12.5 & 14.4 & 1.21 & 0.261 \\
butyric acid & 5.63 & 6.29 & 0.913 & 0.734 \\
valeric acid & 0.59 & 0.64 & 0.152 & 0.788 \\
iso-butyric acid & 0.83 & 0.82 & 0.124 & 0.955 \\
iso-valeric acid & 0.43 & 0.44 & 0.081 & 0.851 \\
succinic acid & 0.00 & 0.00 & - & - \\
total organic acid & 62.4 & 66.8 & 5.98 & 0.474 \\
\hline
\end{tabular}

* 24 calves were included in the dataset; 12 calves received CON and 12 calves received PRO diet, SEM - standard error of the mean
$(P<0.001)$ (Table 1). In the colon, PRO group also had a significantly higher number of yeast cells $(P=0.003)$ and tended to have a lower number of $C$. perfringens than the $\mathrm{CON}$ group $(P=0.064)$.

\section{Concentration of organic acids in the small intestine and colon}

The treatments did not affect the concentrations of any of the measured organic acids in the small intestine or colon (Table 2). Iso-butyric acid, isovaleric acid and $n$-valeric acid were not detectable in the small intestine, while succinic acid was not detectable in the colon of any of the slaughtered calves.

\section{Concentration of biogenic amines the small intestine and colon}

The total concentration and the concentrations of individual biogenic amines in the small intestine were unaffected by the treatments (Table 3).

Table 3. Concentration of biogenic amines in small intestine and colon of rosé veal calves either allocated to a control diet (CON) or a diet supplemented with a product containing yeast (Saccharomyces cerevisiae) and a postbiotic from Lactobacillus acidophilus (PRO) for three weeks*

$\begin{array}{lllll}\text { total biogenic amines } & 141 & 94 & 27.4 & 0.060\end{array}$

${ }^{\star} 24$ calves were included in the dataset; 12 calves received CON and 12 calves received PRO diet, SEM - standard error of the mean

In the colon, the concentrations of individual biogenic amines were not affected by supplementation of the yeast/lactobacillus product, but the total concentration of biogenic amines tended to be lower in PRO group $(P=0.060)$.

\section{Discussion}

To our knowledge, the effects of the yeast/lactobacillus product on the GIT of production animals have not been investigated to date. Thus, only the comparison of the present results with the results of the studies on supplementing SC and postbiotic from Lactobacillus spp. separately is possible. 
The calves received the yeast/lactobacillus product in both MR and concentrate. We expected that the concentrate and MR will have different passage rates in the GIT. The yeast/lactobacillus product in the concentrate will go to the rumen and be more steadily released from here to the lower parts of the GIT whereas the supplement added to the MR will go to the abomasum and be released to the intestine with two major peaks in the hours following the two daily milk feedings. Due to the age of the calves, the concentrate intake was still relatively low at time of slaughter, so the major contribution of the added product was expected to originate from the supplementation in the MR. In addition, CON and PRO groups did also have a similar intake of MR and concentrate, and the average time elapsed from last milk feeding to slaughter was standardized to be the same for both treatment groups. Therefore, the effect of time from last feeding and feed intake on the concentration of metabolites and microbiota between the two treatment groups was expected to be minor. The dirurnal variation in microbiota levels was also expected to be low. However, the concentrations of metabolites could very well fluctuate over the day and the actual presented values need to be seen in relation to the protocol applied herein.

Microbiota in the small intestine and colon. In the current study, the number of Enterobacteriaceae in the small intestine and colon did not differ between the treatments. A similar result was obtained by Mathew et al. (1998) when SC was supplemented at an inclusion level of $1 \mathrm{~g}\left(1 \times 10^{9} \mathrm{CFU} / \mathrm{g}\right) / \mathrm{kg}$ feed to weanling piglets. In contrast, $\mathrm{SC}$ has been found to inhibit the growth of Enterobacteriaceae in the GIT of weaned piglets (Trckova et al., 2014; Zhu et al., 2017). The SC was supplemented at an inclusion level of $5 \mathrm{~g}\left(1 \times 10^{10} \mathrm{CFU} / \mathrm{g}\right) / \mathrm{kg}$ feed in the study by Trckova et al. (2014) and $3 \mathrm{~g}\left(4.3 \times 10^{9} \mathrm{CFU} / \mathrm{g}\right) / \mathrm{kg}$ feed in the study by Zhu et al. (2017). Similarly, postbiotics from Lactobacillus spp. have also been found to decrease the number of Enterobacteriaceae in weaned piglets at an inclusion level of $0.5 \% / \mathrm{kg}$ feed (Loh et al., 2013). For both treatments, the levels of Enterobacteriaceae obtained in the current study lie within the levels found in faecal samples of healthy calves (Schwaiger et al., 2020). This is in good agreement with the fact that none of the calves in the current study received any disease treatment during the three weeks of experiment. Thus, the lack of a treatment effect might be explained by a too low morbidity or by a too low inclusion rate of the product.

Clostridium perfringens is a common member of the intestinal microbiota of calves and can then be generally considered as a commensal (Malmuthuge et al., 2014). However, their uncontrolled multiplication under certain conditions can result in the overproduction of toxins in the GIT (Cho and Yoon, 2014). In the present study, the number of $C$. perfringens in the small intestine was similar in both groups, while PRO calves tended to have a lower number in the colon. Tsai et al. (2016) found a similar effect of the supplementation of $0.2 \% \mathrm{SC} / \mathrm{kg}$ feed on the level of faecal C. perfringens in both sows and piglets. The tendency to lower the number of $C$. perfringens in the colon of the PRO group might be caused by increased competition for nutrients and/or adherence space in the GIT (Mathipa and Thantsha, 2017) as the PRO group also had significantly higher numbers of yeast cells in both small intestine and colon in comparison to CON group. This also indicates that the yeasts are able to survive in the GIT. Similar results in calves were found by Fomenky et al. (2017) when SC was supplemented at the level of $7.5 \times 10^{8} \mathrm{CFU} / 1$ MR and $3 \times 10^{9} \mathrm{CFU} / \mathrm{kg}$ feed.

An increased population of lactic acid bacteria is important for the gastrointestinal health of the host as it contributes to the prevention of pathogen growth (Loh et al., 2013). It was not expected that SC in the yeast/lactobacillus product would affect the number of lactic acid bacteria as in several studies there was no effect. Thus, Fomenky et al. (2017) found no effect of the supplementation of SC $\left(7.5 \times 10^{8} \mathrm{CFU} / 1\right.$ $\mathrm{MR}$ and $3 \times 10^{9} \mathrm{CFU} / \mathrm{kg}$ feed) on the number of total lactobacilli in the small intestine and colon of calves, both prior and after weaning. Similar results were found by Mathew et al. (1998) and Zhu et al. (2019) in the small intestine of weaned piglets. In the studies, $1 \mathrm{~g} \mathrm{SC}\left(1 \times 10^{9} \mathrm{CFU} / \mathrm{g}\right) / \mathrm{kg}$ feed and $3 \mathrm{~g}$ $\mathrm{SC}\left(4.3 \times 10^{9} \mathrm{CFU} / \mathrm{g}\right) / \mathrm{kg}$ feed was supplemented, respectively. However, postbiotics from Lactobacillus spp. have shown to increase the number of lactic acid bacteria in the GIT of weaned piglets at an inclusion rate of $0.3-0.5 \% / \mathrm{kg}$ feed (Thu et al., 2011; Loh et al., 2013). Thus, an increased number of lactic acid bacteria might have been expected due to the presence of postbiotic in the product. However, in the current study, the number of lactic acid bacteria was similar between the treatments in both the small intestine and colon.

Concentration of organic acids in the small intestine and colon. In several studies it was found that SC and postbiotics from Lactobacillus spp., when supplemented separately, can increase the concentration of organic acids in the GIT of pigs (Thu et al., 2011; Loh et al., 2013; Gong et al., 2018). 
Thu et al. (2011) and Loh et al. (2013) found that the inclusion of 0.3 and $0.5 \%$ of postbiotic from Lactobacillus spp. $/ \mathrm{kg}$ feed increased the concentration of organic acids in the GIT of postweaning piglets. Gong et al. (2018) found that the supplementation of $3 \mathrm{~g} \mathrm{SC}\left(1.8 \times 10^{10} \mathrm{CFU} / \mathrm{g}\right) / \mathrm{kg}$ diet increased the concentration of organic acids in the GIT of finisher pigs.

In the present study, we did not detect an effect of the yeast/lactobacillus product on the concentration of organic acids.

Concentration of biogenic amines in the small intestine and colon. In ruminants, biogenic amines originate from dietary sources, e.g. diets with a high content of highly rumen degradable protein, and microbial fermentation in the rumen and colon (Steidlová and Kalač, 2002). In the present study, biogenic amines were measured as indicators of microbial proteolytic fermentation to investigate whether the addition of the yeast/lactobacillus product had an effect on the composition and/or activity of the microbiota. In the small intestine, the amounts of biogenic amines did not differ between the treatments. This implies a similar intake and microbial production of biogenic amines in the rumen. Unfortunately, we have no rumen data from these calves.

The protein that escapes the rumen and ileal digestion can be metabolized by the commensal microbiota in the colon (Steidlová and Kalač, 2002). Enterobacteriaceae and C. perfringens can synthesize putrescine and cadaverine from arginine and lysine, respectively (Dai et al., 2011). Even though the CON group tended to have a higher number of C. perfringens in the colon, the concentrations of putrescine and cadaverine did not differ significantly between the treatments. This complies with the similar number of Enterobacteriacae in the colon. However, the PRO group tended to have a lower total concentration of biogenic amines in the colon. This was due to the accumulated effects of slightly higher amounts of all the individual biogenic amines in the colon of CON group.

\section{Conclusions}

Calves supplemented with the yeast/lactobacillus product had a significantly higher number of yeast cells in both the small intestine and the colon. In addition, the PRO group (supplemented with the product containing the probiotic and postbiotic) also tended to have a lower number of Clostridium perfringens in the colon. The treatments did not affect the concentration of any of the measured organic acids in the gastrointestinal tract. Neither were the concentrations of the individual biogenic amines affected by the treatments; however, PRO group tended to have a lower total concentration of biogenic amines in the colon in comparison to control group.

\section{Acknowledgments}

The study was carried out within the research project 'Robust calves' granted by The Danish Milk Levy Fund and The Danish Cattle Levy Fund. We are indebted to the skilled work done by the private farmer and his staff where the trial took place. The yeast/lactobacillus products were supplied to the farmer free of charge by ChemVet (Silkeborg, Denmark). The authors would also like to thank Thomas Rebsdorf for his technical assistance during sample processing and analysis.

\section{References}

Alugongo G.M., Xiao J., Wu Z., Lil S., Wang Y., Cao Z., 2017. Review: Utilization of yeast of Saccharomyces cerevisiae origin in artificially raised calves. J. Anim. Sci. Biotech. 8, E34-E34, https://doi.org/10.1186/s40104-017-0165-5

Baynes R.E., Dedonder K., Kissell L., Mzyk D., Marmulak T., Smith G., Tell L., Gehring R., Davis J., Riviere J.E., 2016. Health concerns and management of select veterinary drug residues. Food Chem. Toxicol. 88, 112-122, https://doi.org/10.1016/j. fct.2015.12.020

Canibe N., Højberg O., Badsberg J.H., Jensen B.B., 2007. Effect of feeding fermented liquid feed and fermented grain on gastrointestinal ecology and growth performance in piglets. J. Anim. Sci. 85, 2959-2971, https://doi.org/10.2527/jas.2006-744

Cho Y.I., Yoon K.J., 2014. An overview of calf diarrhea - infectious etiology, diagnosis, and intervention. J. Vet. Sci. 15, 1-17, https://doi.org/10.4142/jvs.2014.15.1.1

Dai Z.L., Wu G., Zhu W.Y., 2011. Amino acid metabolism in intestinal bacteria: Links between gut ecology and host health. Front. Biosci. 16, 1768-1786, https://doi.org/10.1093/molehr/gav003

Fomenky B.E., Chiquette J., Bissonnette N., Talbot G., Chouinard Y.P., Ibeagha-Awemu E.M., 2017. Impact of Saccharomyces cerevisiae boulardii CNCMI-1079 and Lactobacillus acidophilus BT1386 on total lactobacilli population in the gastrointestinal tract and colon histomorphology of Holstein dairy calves. Anim Feed Sci. Technol. 234, 151-161, https:// doi.org/10.1016/j.anifeedsci.2017.08.019

Gong Y.L., Liang J.B., Jahromi M.F., Wu Y.B., Wright A.G., Liao X.D., 2018. Mode of action of Saccharomyces cerevisiae in enteric methane mitigation in pigs. Animal 12, 239-245, https://doi. org/10.1017/S1751731117001732

Loh T.C., Thu T.V., Foo H.L., Bejo M.H., 2013. Effects of different levels of metabolite combination produced by Lactobacillus plantarum on growth performance, diarrhoea, gut environment and digestibility of postweaning piglets. J. Appl. Anim. Res. 41, 200-207, https://doi.org/10.1080/09712119.2012.741046

Malmuthuge N., Griebel P.J., Guan L.L., 2014. Taxonomic identification of commensal bacteria associated with the mucosa and digesta throughout the gastrointestinal tracts of preweaned calves. Appl. Environ. Microbiol. 80, 2021-2028, https://doi. org/10.1128/AEM.03864-13 
Martín R., Langella P., 2019. Emerging health concepts in the probiotics field: Streamlining the definitions. Front. Microbiol. 10, 1047-1047, https://doi.org/10.3389/fmicb.2019.01047

Mathew A.G., Chattin S.E., Robbins C.M., Golden D.A., 1998. Effects of a direct-fed yeast culture on enteric microbial populations, fermentation acids, and performance of weanling pigs. J. Anim. Sci. 76, 2138-2145, https://doi.org/10.2527/1998.7682138x

Mathipa M.G., Thantsha M.S., 2017. Probiotic engineering: Towards development of robust probiotic strains with enhanced functional properties and for targeted control of enteric pathogens. Gut Pathog. 9, 1-17, https://doi.org/10.1186/ s13099-017-0178-9

Poulsen A.R., Jonge N., Nielsen J.L., Hojberg O., Lauridsen C., Cutting S.M., Canibe N., 2018. Impact of Bacillus spp. spores and gentamicin on the gastrointestinal microbiota of suckling and newly weaned piglets. PLoS ONE 13, e0207382, https:// doi.org/10.1371/journal.pone.0207382

Schwaiger K., Storch J., Bauer C., Bauer J., 2020. Development of selected bacterial groups of the rectal microbiota of healthy calves during the first week postpartum. J. Appl. Microbiol. 128, 366-375, https://doi.org/10.1111/jam.14484

Steidlová Š., Kalač P., 2002. Levels of biogenic amines in maize silages. Anim. Feed Sci. Technol. 102, 197-205, https://doi. org/10.1016/S0377-8401(02)00217-1

Thorsteinsson M., Vestergaard M., 2020. Performance and health of young rosé veal calves supplemented with yeast (Saccharomyces cerevisiae) and a postbiotic from Lactobacillus acidophilus. J. Anim. Feed Sci. 29, 115-124, https://doi.org/10.22358/jafs/124040/2020
Thu T.V., Loh T.C., Foo H.L., Yaakub H., Bejo M.H., 2011. Effects of liquid metabolite combinations produced by Lactobacillus plantarum on growth performance, faeces characteristics, intestinal morphology and diarrhoea incidence in postweaning piglets. Trop. Anim. Health Prod. 43, 69-75, https://doi. org/10.1007/s11250-010-9655-6

Trckova M., Faldyna M., Alexa P., Zajacova Z.S., Gopfert E., Kumprechtova D., Auclair E., D'Inca R., 2014. The effects of live yeast Saccharomyces cerevisiae on postweaning diarrhea, immune response, and growth performance in weaned piglets. J. Anim. Sci. 92, 767-774, https://doi. org/10.2527/jas.2013-6793

Tsai T.C., Kim H.J., Wang X., Bass B.E., Frank J.W., Maxwell C.V., 2016. Effect of Saccharomyces cerevisiae fermentation product supplementation in late gestation and lactation on sow and litter performance, milk components, and fecal Clostridium perfringens. J. Anim. Sci. 94, 134-134, https:// doi.org/10.2527/msasas2016-286

Wegh C.A.M., Geerlings S.Y., Knol J., Roeselers G., Belzer C., 2019. Postbiotics and their potential applications in early life nutrition and beyond. Int. J. Mol. Sci. 20, e4673, https://doi. org/10.3390/ijms20194673

Williams N.T., 2010. Probiotics. Am. J. Health-Syst. Pharm. 67, 449-458, https://doi.org/10.2146/ajhp090168

Zhu C., Wang L., Wei S., Chen Z., Ma X., Zheng C., Jiang Z., 2017. Effect of yeast Saccharomyces cerevisiae supplementation on serum antioxidant capacity, mucosal slgA secretions and gut microbial populations in weaned piglets. J. Integr. Agri. 16, 2029-2037, https://doi.org/10.1016/S2095-3119(16)61581-2 